\title{
Polar Mesoscale Cyclones in the Northeast Atlantic: Comparing Climatologies from ERA-40 and Satellite Imagery
}

\author{
Alan Condron and Grant R. BigG \\ Department of Geography, University of Sheffield, Sheffield, United Kingdom \\ IAN A. RENFREW \\ School of Environmental Sciences, University of East Anglia, Norwich, United Kingdom
}

(Manuscript received 13 June 2005, in final form 29 September 2005)

\begin{abstract}
Polar mesoscale cyclones over the subarctic are thought to be an important component of the coupled atmosphere-ocean climate system. However, the relatively small scale of these features presents some concern as to their representation in the meteorological reanalysis datasets that are commonly used to drive ocean models. Here polar mesocyclones are detected in the 40-Year European Centre for Medium-Range Weather Forecasts (ECMWF) Re-Analysis dataset (ERA-40) in mean sea level pressure and 500-hPa geopotential height, using an automated cyclone detection algorithm. The results are compared to polar mesocyclones detected in satellite imagery over the northeast Atlantic, for the period October 1993September 1995. Similar trends in monthly cyclone numbers and a similar spatial distribution are found. However, there is a bias in the size of cyclones detected in the reanalysis. Up to $80 \%$ of cyclones larger than $500 \mathrm{~km}$ are detected in MSL pressure, but this hit rate decreases, approximately linearly, to $\sim 40 \%$ for $250-\mathrm{km}$-scale cyclones and to $\sim 20 \%$ for $100-\mathrm{km}$-scale cyclones. Consequently a substantial component of the associated air-sea fluxes may be missing from the reanalysis, presenting a serious shortcoming when using such reanalysis data for ocean modeling simulations. Eight maxima in cyclone density are apparent in the mean sea level pressure, clustered around synoptic observing stations in the northeast Atlantic. They are likely spurious, and a result of unidentified shortcomings in the ERA-40 data assimilation procedure.
\end{abstract}

\section{Introduction}

Polar mesocyclones are a mesoscale (typically 100 $1000 \mathrm{~km}$ in diameter) maritime weather system with a relatively short life time of 3-36 h (see, e.g., Businger and Reed 1989; Rasmussen and Turner 2003; Renfrew 2003). The most intense form of polar mesoscale cyclones make up a small group referred to as polar lows, in which near-surface wind speeds exceed $15 \mathrm{~m} \mathrm{~s}^{-1}$. With the advent of satellite imagery it has become apparent that polar mesocyclones are frequent in both hemispheres of the subarctic Atlantic and Pacific Oceans (see, e.g., Businger 1987; Carleton and Carpenter 1990; Harold et al. 1999a). In the North Atlantic, vortices are commonly observed over both the Labra-

Corresponding author address: Dr. Alan Condron, Department of Geography, University of Sheffield, Winter Street, Sheffield S10 2TN, United Kingdom.

E-mail: Alan.Condron@sheffield.ac.uk dor Sea (Moore et al. 1996; Rasmussen et al. 1996) and the Greenland Sea (Harold et al. 1999a; Shapiro et al. 1987; Nordeng and Rasmussen 1992) in regions where intense buoyancy loss due to heat exchange with the overlying atmosphere and sea ice formation are important for initiating localized violent open-ocean convection that mixes the surface waters to great depths, given suitable preconditioning (Marshall and Schott 1999; Wadhams et al. 2002). The water mass modifications that occur in these regions due to convection appear to be the main Northern Hemisphere control on the strength of the thermohaline circulation, and thus have far-reaching effects on the climate system (Marshall and Schott 1999).

In the North Atlantic, the most vigorous polar mesoscale depressions report wind speeds in excess of 60 $\mathrm{kt}\left(\sim 31 \mathrm{~m} \mathrm{~s}^{-1}\right)$, and generally occur during cold arctic air outbreaks (Rasmussen 1985). These high wind speeds and the large air-sea temperature contrasts can locally enhance the air-sea exchange of heat, moisture, 
and momentum, providing these systems with the potential to reduce surface buoyancy and influence convective overturning. Research aircraft observations from a polar mesocyclone over the Norwegian Sea reveal extremely large total turbulent (latent plus sensible) heat fluxes out of the ocean into the atmosphere, exceeding $1000 \mathrm{~W} \mathrm{~m}^{-2}$, and are on a par with heat fluxes found within mature tropical cyclones (Shapiro et al. 1987).

Atmospheric reanalysis datasets are frequently used to drive ocean models, for example, Hakkinen (1995) used wind field data from the National Center for Atmospheric Research (NCAR) reanalysis project as part of the forcing for a fully prognostic Arctic ice-ocean model. The importance of localized wind conditions in controlling the occurrence of deep convection in the Greenland Sea gyre was highlighted by showing that enhanced deep convection occurred in this region during periods with higher numbers of extreme wintertime wind events. The accurate representation of polar mesocyclonic vortices in meteorological reanalysis datasets is therefore vital if we are to confidently use these datasets in such climate forcing experiments. To determine the extent to which polar mesocyclones are captured in such datasets, here we compare the number and spatial distribution of vortices in the latest 40-Year European Centre for Medium-Range Weather Forecasts (ECMWF) Re-Analysis (ERA-40) with mesocyclonic cloud features observed in satellite imagery over the northeast Atlantic from October 1993 to September 1995 (as documented in Harold et al. 1999a). The presence of a cyclonic cloud signature in the satellite imagery suggests that circulation is occurring in the atmosphere, with the potential to influence the underlying ocean. Cloud signatures that are not found to have a cyclonic circulation in the reanalysis may either be too small and/or weak to be resolved or may be confined to the upper level of the atmosphere and therefore will not have a surface signature. The satellite imagery highlights a region of intense mesocyclonic activity over the convectively important Greenland Sea, in a location where relatively few synoptic-scale cyclones penetrate (Harold et al. 1999a). Nevertheless, the short-lived nature of many of these systems, and their occurrence in remote areas may cause them to fall outside of the relatively sparse synoptic weather station network that exists away from land in the North Atlantic sector, and therefore not be assimilated into the reanalysis. This paper quantifies the number of polar mesocyclones captured in the reanalysis using an automated procedure to yield consistent and repeatable results. As far as we know there has been no attempt to objectively identify polar mesocyclones in analysis or reanalysis data, and so we believe this study represents the first such objective climatology using this data source. In the next section we describe the datasets used. This is followed by a presentation and discussion of the results in sections 3 and 4. Section 5 summarizes the results and presents conclusions.

\section{Datasets}

\section{a. Satellite data}

A database of polar mesocyclones was compiled using Advanced Very High Resolution Radiometer (AVHRR; 11- $\mu \mathrm{m}$ infrared) satellite imagery by Harold et al. (1999a), at a spatial resolution of $6 \mathrm{~km}$ making it perfectly adequate for observing polar mesocyclones. Data were collected from 1 October 1993 to 30 September 1995 with an average of five images per day examined (no day without an image was recorded). It was subjectively analyzed for all cloud systems with a cyclonic curvature (spiral or comma shaped) over the northeast Atlantic; a thorough review of the interpretation and classification of mesocyclones from satellite imagery is given in Carleton (1985). Due to the typical satellite pass coverage, observations were restricted to $50^{\circ}-80^{\circ} \mathrm{N}, 50^{\circ} \mathrm{W}-50^{\circ} \mathrm{E}$, and if a system moved permanently over land it was ignored from that point on (i.e., only maritime mesocyclones were considered), although mesocyclones passing over the United Kingdom and Iceland were considered. A total of 7792 cyclonic cloud patterns were observed from 3735 images. However, $31 \%$ of these cloud patterns were observed only once, either because they were short lived or because the cyclone moved out of the study area. As far as we are aware this dataset is the only climatology of polar mesocyclones in the northeast Atlantic. Existing climatologies have typically examined only the intense subset of polar lows, and often focus only on those systems making landfall (Lystad 1986; Wilhelmsen 1985). Harold et al. (1999a) make no attempt to distinguish between polar mesocyclones and the polar lows or those that only make landfall, thus a large number of systems are identified in their study.

In this study, we restrict the Harold et al. (1999a) dataset to the 5370 mesoscale cloud patterns observed in more than one satellite image, as these are the most likely to significantly impact upon the ocean and to be recorded by the synoptic network, and thus be assimilated into the reanalysis. Note, however, that this number is different from the 2595 individual multisighted cyclones given in Harold et al. (1999a) as we make no attempt to track cyclones from one time period to the next. Clearly a cyclone may persist for several satellite images and thus would be counted as the same system 
if tracked. By only considering multisighted cyclones, cloud signatures that may have been misclassified as polar mesocyclones, but were in fact nondeveloping minor vortices, or simply fields of cumulus that appeared to have a cyclonic cloud pattern, will be neglected. Most of the polar mesocyclones observed formed and decayed within one day and traveled only relatively short distances. They had a continuous spectrum of diameters, but with a distribution skewed toward smaller diameters, illustrated by a modal size of $100-150 \mathrm{~km}$ (Harold et al. 1999a), and in agreement with observations quoted by Carleton (1985). Cyclone activity was observed to be highest during the winter period (January, February, and March) when the greatest temperature gradients occur between the land and the ocean, leading to the potential for strong baroclinic zones along their boundary (Harold et al. 1999a).

\section{b. ERA-40 data}

To compare with the subjective satellite climatology, an objective cyclone climatology from the ECMWF numerical weather prediction reanalysis, ERA40, was compiled. The ERA-40 data assimilation system uses the Integrated Forecasting System (IFS) developed jointly by ECMWF and Météo-France to produce a comprehensive set of global analyses describing the state of the atmosphere in three dimensions at any one time (Simmons and Gibson 2000). The assimilating model has T159 spectral resolution in the horizontal and 60 levels in the vertical, and the data covers a 45-yr period from September 1957 to August 2002 with data available every $6 \mathrm{~h}$. The ECMWF ERA-40 data were available to us on a reduced Gaussian grid with 80 grid points aligned along the Greenwich Meridian from the equator to the Pole, giving a north-south grid spacing of $1.125^{\circ}(\sim 125 \mathrm{~km})$. In the east-west direction the gridpoint resolution varies with latitude; in the Tropics 320 grid points produce a grid spacing of $125 \mathrm{~km}$, while at $60^{\circ}$ and $70^{\circ} \mathrm{N}$ (in our study region) the gridpoint spacing gives a resolution of $\sim 111$ and $\sim 106 \mathrm{~km}$, respectively. The smallest feature the ERA-40 spectral model can represent is given by the spectral truncation limit, T159, or approximately $125 \mathrm{~km}$ (Simmons and Gibson 2000). However, for an accurate representation and for the advection of features in a spectral model, it is suggested that they must be $2-4$ times this smallest scale (e.g., Pielke 1991; Laprise 1992). Hence, one might expect some representation of features of scale $\sim 125-500 \mathrm{~km}$ and accurate representation of features of scale $>500 \mathrm{~km}$.

The 2-yr period of comparison represents a period of rather high quality ERA-40 data since after 1991 the reanalysis benefits from the assimilation of remotely sensed Special Sensor Microwave Imager (SSM/I) data providing ocean wind speed (at $10 \mathrm{~m}$ ), water vapor, cloud water, and rain rate, as well as European Remote Sensing satellite (ERS) scatterometer wind data available at a $50 \mathrm{~km} \times 50 \mathrm{~km}$ resolution (Simmons and Gibson 2000), thus increasing the chances of capturing mesocyclonic features.

Two fields are used in this study: mean sea level (MSL) pressure and 500-hPa geopotential height. Businger (1985) found that the intense polar mesocyclones known as polar lows in the Norwegian and Barents Sea were associated with negative height anomalies in the 500-hPa geopotential height field. Similarly, Turner and Thomas (1994) showed polar mesocyclones in the Bellingshausen-Weddell region of Antarctic to be associated with anomalies of $-5.3 \mathrm{dm}$ and $-0.5 \mathrm{hPa}$ in the 500-hPa height and MSL pressure fields, respectively.

\section{c. Cyclone detection}

It is common to detect cyclones by finding a minimum in the MSL pressure field with respect to the surrounding grid points (see, e.g., Terry and Atlas 1996). However, this method favors larger deeper systems, and may exclude weaker or smaller mesocyclones (Sinclair 1994; Leonard et al. 1999). An improvement in detecting smaller cyclones can be made using geostropic relative vorticity rather than a pressure minimum (Sinclair 1994) and furthermore vorticity centers correlate better to the center of cyclones observed on satellite imagery than do pressure minima (Sinclair 1994). It is known that polar mesocyclones are usually associated with a maximum in strong positive relative vorticity (see, e.g., Businger 1985; Shapiro et al. 1987). Indeed in a study of polar mesocyclones outbreaks over the northeast Atlantic Hewson et al. (1995) considered each system to exist at a given time only if a localized maximum in the surface geostrophic relative vorticity was present.

Here we use the cyclone detection algorithm derived by Murray and Simmonds (1991) to locate polar mesocyclones in MSL pressure and 500-hPa geopotential height fields in the ERA-40 analysis. In this cyclonefinding scheme, vortices are identified by locating a local maximum in the Laplacian of the pressure field (i.e., $\left.\nabla^{2} p\right)$ or geopotential height $\left(\nabla^{2} z ;\right.$ Fig. 1). This approach is similar to that in which lows are identified as maxima in vorticity (see, e.g., Sinclair 1994), as the Laplacian of the pressure is proportional to the geostrophic relative vorticity $\left(\xi_{g}\right)$ :

$$
\xi_{g}=\frac{1}{\rho f} \nabla^{2} p,
$$




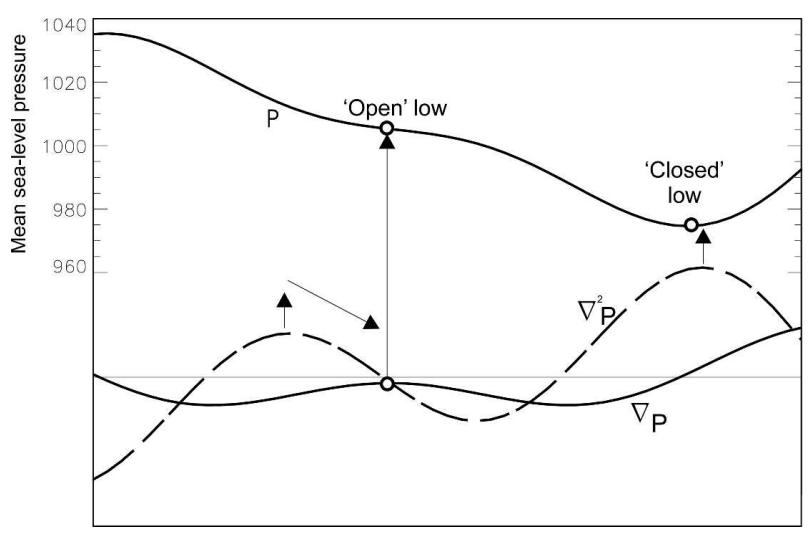

FIG. 1. A cross section of pressure and its derivatives showing how the maxima in $\nabla^{2} p$ may be used as a starting point to locate both "open" and "closed" depressions. A closed low will often lie fairly close to its $\nabla^{2} p$ maximum, while an open low is associated with the closest absolute minimum in $\nabla p$. Modified from Fig. 5 of Murray and Simmonds (1991).

where

$$
\xi_{g}=\frac{\partial v_{g}}{\partial x}-\frac{\partial u_{g}}{\partial y}
$$

and $u_{g}$ and $v_{g}$ are the geostrophic wind components, $\rho$ is the density, $f$ is the Coriolis parameter, and $p$ is the pressure. Hence, this technique is well suited for locating polar mesocyclones in gridded datasets by identifying the smallest cyclonic vortices represented in the reanalysis data. A reliance on locating cyclones based purely on a minimum in the MSL pressure field will exclude smaller systems potentially of mesocyclonic scale from being detected.

Upon locating a maximum in the Laplacian of the pressure (or geopotential height) field, a search is carried out in this region to determine the type of cyclone center. Two different types of center are identified; a "closed" center is identified if a pressure minimum is nearby to the Laplacian maximum (usually within one grid spacing). Such a center would possess a region of closed isobars on a synoptic chart for some finite contour interval. An "open" depression is identified by locating the minimum in the magnitude of the pressure gradient $(|\nabla p|)$ closest to the maximum in $\nabla^{2} p$. Such a center is not associated with a minimum in the pressure field itself, and as such these centers could not be identified by looking for closed contours on synoptic charts. Thus, an open center might correspond to a small weak cyclone, potentially of mesoscale size masked by a larger-scale system, or a trough. In this study, no attempt is made to track the systems from one time period to the next.

\section{d. Dataset comparison procedure}

To facilitate direct comparison between the number of vortices detected in the satellite imagery and those in the reanalysis data, we restrict both datasets to include the same number of temporal observations and the same spatial region. This is achieved by selecting the nearest satellite pass to each 6-hourly reanalysis time. If no satellite pass can be located within $\pm 3 \mathrm{~h}$ of the reanalysis time then cyclones from this time period are ignored. Consecutive satellite times are then examined until a suitable time is located. On selecting an appropriate satellite pass, vortices in the reanalysis data are restricted to the area observed by the satellite (Fig. 2) by postprocessing the reanalysis data at Dundee Satellite Receiving Station to remove depressions outside of the observable area. Frequently more than one satellite image was available within $\pm 3 \mathrm{~h}$ of the reanalysis time. When this occurs only the closest satellite pass time to the reanalysis time is used. All other satellite passes, and the vortices located by Harold et al. (1999a) within the $\pm 3 \mathrm{~h}$ time interval, are disregarded. Polar mesocyclones over land are also removed from the reanalysis data as they were neglected in the Harold et al. (1999a) climatology. Overall, a total of 1595 satellite and reanalysis times were compared, with a satellite pass found on average $\pm 1 \mathrm{~h} 25 \mathrm{~min}$ of the reanalysis time, with a standard deviation of $56 \mathrm{~min}$. Due to the satellite orbits, the majority of the comparisons were made between 0600 and 1800 UTC, with only $7 \%$ of reanalyzed data at 0000 UTC having a satellite pass within $\pm 3 \mathrm{~h}$. In fact, no satellite passes were made over the study domain between 2200 and 0000 UTC during the 2-yr period.

Distances between all satellite and reanalyzed systems are calculated following the successful match of a satellite pass to a time in the reanalysis data. If the distance between a cloud vortex and a reanalyzed depression is less than the radius of the cloud vortex (as measured from the satellite image by Harold et al. 1999a) then the cloud system is deemed to have a detectable signature in the reanalysis data. We expand the radius of each cloud vortex to account for propagation away from its initial recorded position over the elapsed time between the reanalysis and the satellite pass. A speed of $15 \mathrm{~m} \mathrm{~s}^{-1}$ in any direction is assumed, which is consistent with observed speeds of mesocyclone movement (see, e.g., Hewson et al. 1995; Shapiro et al. 1987). When two (or more) vortices in the reanalysis data are associated with the same cloud feature only one of the reanalysis systems will be classified as belonging to the cloud signature. In this instance, the choice of cyclone is made based on its strength and distance from the cloud 

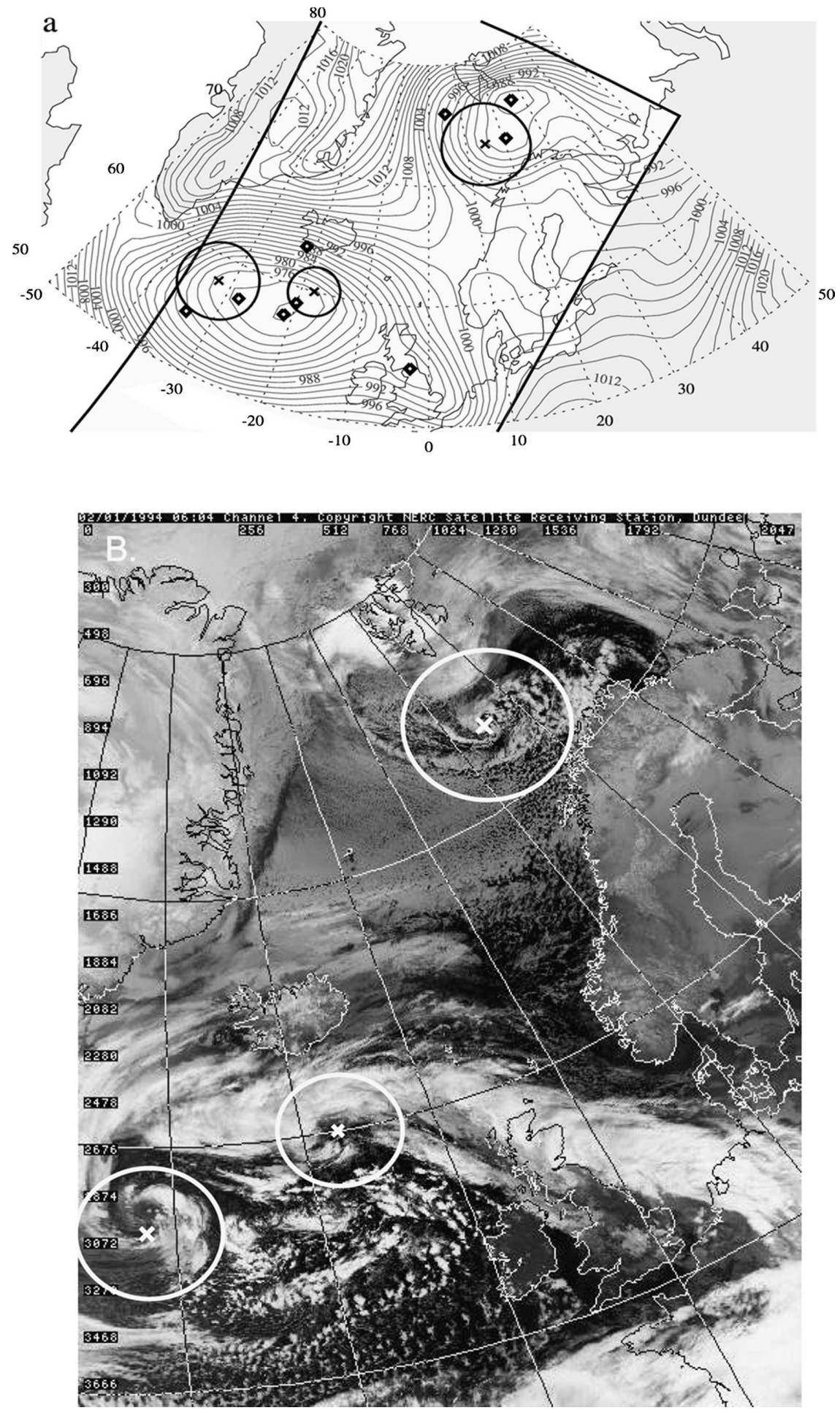

FIG. 2. (a) MSL pressure field at 0600 UTC 2 Jan 1994 from ERA-40. The contour interval is $2 \mathrm{hPa}$. Nine cyclones identified in the reanalysis are marked by black diamonds, and the three multisighted mesocyclones in the satellite climatology are marked by circles with a cross at the center. The outline of the satellite pass is marked by a bold line, with reanalyzed cyclones outside of this area removed. (b) The closest AVHRR satellite image taken at 0604 UTC 2 Jan 1994, showing the three multisighted mesocyclones. (Image courtesy of the NERC Satellite Receiving Station, University of Dundee, Dundee, United Kingdom.) 
vortex; vortices with a closed pressure contour in the reanalysis and those closest to the center of the cloud vortex are preferentially selected. Figure 2 illustrates the matching procedure. The MSL pressure field for 0600 UTC 2 January 1994 depicts two synoptic cyclones in the northeast Atlantic, the largest of which is located south of Iceland with a central pressure of $973 \mathrm{mb}$, and the second situated just southeast of Spitzbergen, Norway, with a central pressure of $981 \mathrm{mb}$. The locations of these two closed cyclones along with a further seven open vortices found by the cyclone algorithm are indicated on the chart. The closest corresponding satellite observation was available $4 \mathrm{~min}$ after the reanalysis at 0604 UTC 2 January 1994, and reveals three polar mesocyclones, two of which are located in the central northeast Atlantic at approximately $56^{\circ} \mathrm{N}, 33^{\circ} \mathrm{W}$ and $60^{\circ} \mathrm{N}, 18^{\circ} \mathrm{W}$, while the third is positioned just south of Spitzbergen at around $73^{\circ} \mathrm{N}, 15^{\circ} \mathrm{W}$. Cyclones in the reanalysis were restricted to the area observed during the satellite pass as well as being removed over land, which resulted in the removal of nine additional vortices. In Fig. 2 all three cyclonic cloud features identified are concurrent with a circulation signature at MSL pressure, as each system occurs in the vicinity of a reanalyzed cyclone. In this example, it is also worth noting that all of the cloud systems correspond to open vortices, and therefore had cyclones been detected simply as a local minimum in pressure, no circulation signature would have been prescribed to each cloud system making them appear absent from the reanalysis.

To optimize the detection of polar mesocyclones, the algorithm of Murray and Simmonds (1991) was tuned to ensure that the largest number of cloud vortices were associated with cyclones in the reanalysis pressure fields. It is common to apply a spatial smoothing over a constant radius to pressure data prior to searching for cyclones to remove weaker vortices (Sinclair 1994; Murray and Simmonds 1991; Simmonds et al. 1999). For the detection of synoptic-scale depressions Simmonds et al. (1999) indicate that a spatial smoothing of $2^{\circ}$ is satisfactory. However, large cyclones that contain several closely spaced mesoscale vortices that may individually be meteorologically significant can become merged into a single synoptic vortex as a result of smoothing (Sinclair 1997). We find that spatially smoothing the reanalysis data significantly reduces the likelihood of associating a satellite-observed mesocyclone with a vortex in the pressure data, and as such no smoothing is made.

Cyclone strengths in the MSL pressure and 500-hPa geopotential height fields are calculated by averaging the central Laplacian of the pressure maxima over a $1^{\circ}$ radius. Sensitivity tests were carried out to establish how increasing the Laplacian acceptance strength threshold ( $\left.L_{\text {threshold }}\right)$ affected their association with polar mesocyclonic cloud vortices. For MSL pressure, the removal of vortices with Laplacian strengths below 3 $\mathrm{hPa}(\mathrm{deg})^{-2}$ [i.e., $L_{\text {threshold }}$ set to $3 \mathrm{hPa}(\mathrm{deg})^{-2}$ ] was found to have the most significant influence, while above this threshold little sensitivity was observed. At the 500-hPa geopotential height level, removing cyclones below a Laplacian strength of $15 \mathrm{gpm}(\mathrm{deg})^{-2}$ had the strongest influence on their association with cloud vortices in the satellite imagery, while above this little sensitivity was once again observed. In the following sections we present results with the $L_{\text {threshold }}$ set at $0,1,2,3 \mathrm{hPa}(\mathrm{deg})^{-2}$ and $0,5,10,15 \mathrm{gpm}(\mathrm{deg})^{-2}$ for MSL pressure and 500-hPa geopotential height, respectively.

\section{Results}

\section{a. MSL pressure}

By allowing all maxima in the Laplacian of the MSL pressure field to be classed as cyclones [i.e., $L_{\text {threshold }}=$ $0 \mathrm{hPa}(\mathrm{deg})^{-2}$ ], $48 \%$ of cloud vortices identified in the satellite imagery can be associated with features in the pressure field over the 2-yr period, and thus can be thought of as polar mesocyclones. In total, $11383 \mathrm{cy}-$ clones were identified in the reanalyzed climatology, which is on average 3.7 times higher than the number observed in the satellite imagery. Nevertheless, the monthly numbers of satellite and reanalyzed vortices both show a very similar trend over the 2 -yr period, with a calculated correlation coefficient of 0.80 that is statistically significant at the $99 \%$ confidence interval (Fig. 3). During the winter period, monthly cyclone activity peaks with 174 and 593 vortices in the satellite and reanalysis climatologies, respectively, compared to only 87 and 359 vortices during the summer months, respectively, when averaged over both seasons. There is a general decline in cyclone activity in both climatologies during the 2-yr period, which is highlighted by a linear regression being placed through both climatologies in Fig. 3, although only the decline in the satellite climatology is found to be statistically significant. In spite of the decline in the reanalysis climatology being shallower than the satellite analysis, both climatologies show a decrease of around 90 cyclones month ${ }^{-1}$ over the 2-yr period. A separate examination of open and closed lows in the reanalysis climatology reveals that the decline is restricted to those systems that are open (Fig. 4). The open lows represent the smaller subsynoptic systems compared to the closed lows, with modal class sizes of 150 - and $450-\mathrm{km}$ diameter, respectively. As shown in Fig. 4b, this observation is consistent with 

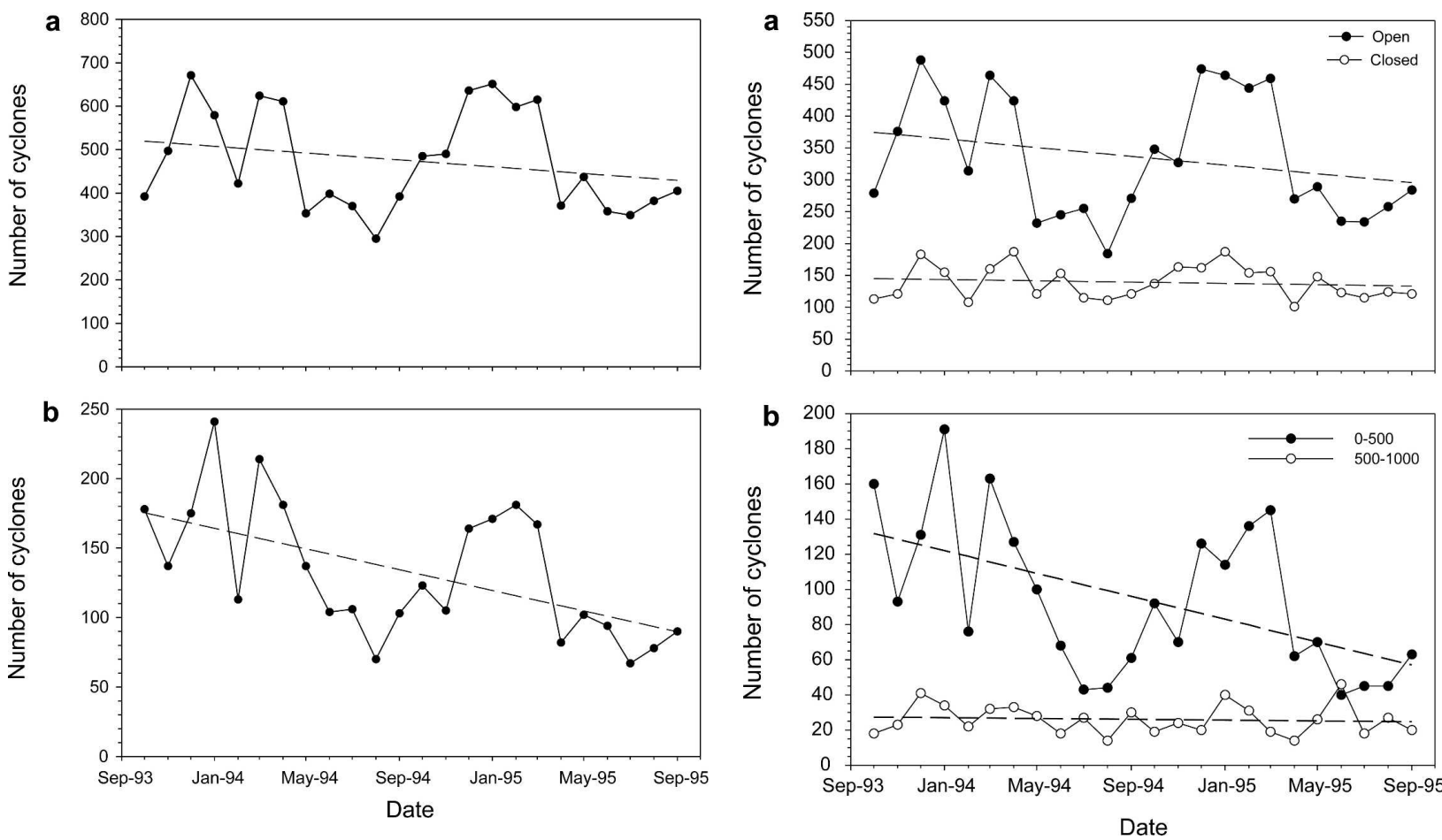

FIG. 3. The monthly number of vortices detected in (a) ECMWF MSL pressure and (b) satellite imagery from October 1993 to September 1995. The dashed black line shows the linear regression trend over the two years, and highlights the decline in cyclone activity.

the satellite climatology, where it is the small cyclones less than $500 \mathrm{~km}$ in diameter that predominantly decline from October 1993 to September 1995.

The removal of weak reanalyzed cyclones has the overall effect of reducing the total number of systems identified in the MSL pressure data over the $2 \mathrm{yr}$ (Table 1). Setting the $L_{\text {threshold }}$ to $1 \mathrm{hPa}(\mathrm{deg})^{-2}$ results in a reduction in the number of satellite vortices with a reanalyzed depression to $44 \%$, while setting $L_{\text {threshold }}$ to 3 $\mathrm{hPa}(\mathrm{deg})^{-2}$ reduces this to $25 \%$. It is interesting to note that despite a reduction in overall numbers, the general pattern in monthly cyclone activity over the $2 \mathrm{yr}$ remains fairly consistent, and continues to resemble the satellite climatology (Fig. 5), with correlation coefficients staying around 0.8 (Table 1). The highest correlation $(r=0.82)$ is noted when $L_{\text {threshold }}$ is set to $1 \mathrm{hPa}$ $(\mathrm{deg})^{-2}$, although only a slightly lower correlation coefficient is obtained when $L_{\text {threshold }}$ is set to $2 \mathrm{hPa}$ $(\mathrm{deg})^{-2}$. At this strength threshold the total number of cyclones is 4176, and is approaching the 3004 observed from satellite imagery.

\section{1) Cyclone distribution}

Cyclone density is used to show the spatial distribution of vortices in the northeast Atlantic in this study.

FIG. 4. The monthly number of (a) open and closed cyclones identified in MSL pressure considering all Laplacian maxima $\left(\nabla^{2} p\right)$ to be cyclones, and (b) vortices detected in satellite imagery with diameters of 0-500 and 500-1000 km from October 1993 to September 1995. The dashed black lines show the linear regression trends for the two years.

This is defined as the number of cyclonic cloud or reanalyzed centers within a $200-\mathrm{km}$ radius centered on each reanalysis grid point. As such, the density calculation can be approximately thought of as the number of features per $125000 \mathrm{~km}^{2}$. Over the computational domain $\left(50^{\circ}-80^{\circ} \mathrm{N}, 50^{\circ} \mathrm{W}-50^{\circ} \mathrm{E}\right)$ the grid spacing means that a given cyclone may be counted at several adjacent grid points. This method of calculating cyclone densities is similar to that used in Sinclair (1994) in which the number of cyclone centers falling within $555 \mathrm{~km}\left(5^{\circ}\right.$ latitude) of each grid point was calculated. The density distribution of polar mesocyclones identified from satellite imagery and in MSL pressure data for the 2-yr period is shown in Fig. 6. From the satellite imagery, the majority of cyclones are detected between $60^{\circ}-77^{\circ} \mathrm{N}$, $20^{\circ} \mathrm{E}-20^{\circ} \mathrm{W}$, with a maxima observed at $\sim 73^{\circ} \mathrm{N}, 5^{\circ} \mathrm{E}$ over the Greenland-Norwegian Sea where cyclone densities are over 150 per $125,000 \mathrm{~km}^{2}$. Over the remaining northeast Atlantic cyclone densities are around 25-50. During the summer, cyclone densities significantly decline over the entire northeast Atlantic, with no cluster of activity over the GreenlandNorwegian Sea (not shown). 
TABLE 1. A comparison of cyclone statistics from October 1993 to September 1995 for MSL pressure and 500-hPa height with satellite-observed cyclones. Results are tabulated for different cyclone strengths as determined by the $L_{\text {threshold }}$. Both the temporal and spatial correlation coefficients are given as $r$ values. The temporal correlations are derived from a Pearson product moment correlation coefficient (Shaw and Wheeler 2000) between monthly reanalyzed and satellite-observed cyclone numbers over the two years, while the spatial correlations are derived from the covariance between the total numbers of reanalyzed and satellite-observed cyclones over the two years. "SR hits" are the percentages of satellite-detected cyclones with a reanalyzed signature, while "RS hits" are the percentages of reanalyzed cyclones with a satellite-detected feature.

\begin{tabular}{lcccccc}
\hline \hline \multicolumn{1}{c}{ Dataset } & $L_{\text {threshold }}$ & $\begin{array}{c}\text { No. of } \\
\text { cyclones }\end{array}$ & $\begin{array}{c}\text { Temporal } \\
\text { correlation coef }\end{array}$ & $\begin{array}{c}\text { Spatial } \\
\text { correlation coef }\end{array}$ & SR hits (\%) & RS hits (\%) \\
\hline Satellite climatology & & 3004 & & & & \\
MSL pressure & 0 & 11383 & 0.80 & 0.75 & 48.2 & 12.9 \\
& 1 & 7822 & 0.82 & 0.82 & 44.4 & 18.4 \\
& 2 & 4176 & 0.82 & 0.86 & 35.0 & 29.5 \\
& 3 & 2128 & 0.79 & 0.85 & 35.0 & 14.3 \\
500-hPa geopotential height & 0 & 7378 & 0.80 & 0.86 & 34.3 & 16.5 \\
& 5 & 6306 & 0.80 & 0.86 & 28.5 & 28.3 \\
& 10 & 4553 & 0.77 & & & 28.5 \\
\end{tabular}

An overall total of 11383 cyclone centers can be found in the MSL pressure reanalysis data over the northeast Atlantic when all maxima in the Laplacian of the MSL pressure field are classed as cyclones (Fig. 6b). The average density distribution across the study region is 40 cyclones per $125000 \mathrm{~km}^{2}$, while the density distribution reveals the highest cyclonic activity to occur east of $30^{\circ} \mathrm{W}$, where cyclone densities are typically 150 200 per $125000 \mathrm{~km}^{2}$. Within this domain, eight regions of extremely high cyclone occurrence are noticed. They are located at $77^{\circ} \mathrm{N}, 10^{\circ} \mathrm{E}$ to the west of Spitzbergen, over Jan Mayen Island $\left(72^{\circ} \mathrm{N}, 10^{\circ} \mathrm{W}\right)$, off the west coast of Norway $\left(68^{\circ} \mathrm{N}, 15^{\circ} \mathrm{E}\right.$ and $\left.65^{\circ} \mathrm{N}, 5^{\circ} \mathrm{E}\right)$, over and to the south of Iceland $\left(\sim 63^{\circ} \mathrm{N}, 20^{\circ} \mathrm{W}\right)$, over the United Kingdom at $57^{\circ} \mathrm{N}, 2^{\circ} \mathrm{W}$ and $52^{\circ} \mathrm{N}, 0^{\circ}$, and eastern Ireland at $53^{\circ} \mathrm{N}, 6^{\circ} \mathrm{W}$. It is interesting to note that these features persist when different-sized radius circles are used to construct the density field maps, and also when the search radius is scaled to account for the latitudinal reduction in grid spacing in the zonal direction toward the Pole (not shown). However, despite an apparent lack of visual similarity with the satellite-observed cyclones there is a high spatial correlation between the two data fields $(r=0.75)$, suggesting the general structure is similar.

As cyclones are screened out based on their Laplacian strength, the eight maxima over the northeast Atlantic are gradually removed, suggesting these maxima comprise fairly "weak" cyclones (Figs. 6c-e). Setting the $L_{\text {threshold }}$ equal to $1 \mathrm{hPa}(\mathrm{deg})^{-2}$, the eight maxima are still apparent but less distinct, especially over the United Kingdom and Ireland. However, the maxima over Iceland, at Jan Mayen Island, along the Norwegian coast and to the west of Spitzbergen are still distinct. When the $L_{\text {threshold }}$ equals $2 \mathrm{hPa}(\mathrm{deg})^{-2}$, the cyclonic maxima are only distinct over Jan Mayen Island and to the west of Spitzbergen with counts of 160 and 200 cyclones per $125000 \mathrm{~km}^{2}$, respectively. Removing weaker strength cyclones results in an improved spatial correlation with the Harold et al. (1999a) data (Table 1). The highest correlation is obtained when $L_{\text {threshold }}$ is set to $2 \mathrm{hPa}(\mathrm{deg})^{-2}(r=0.86)$. At this strength monthly cyclone numbers closely correspond to those observed in the satellite imagery. Increasing the $L_{\text {threshold }}$ to $3 \mathrm{hPa}(\mathrm{deg})^{-2}$ results in most of the cyclone maxima being removed.

\section{2) Characteristics of SYSTEMS FOUnd IN MSL PRESSURE ERA-40}

There is a bias toward the size of the cloud system that can be detected, with larger cloud systems more frequently having a detectable signature in the MSL pressure reanalysis data (Fig. 7). When all Laplacian

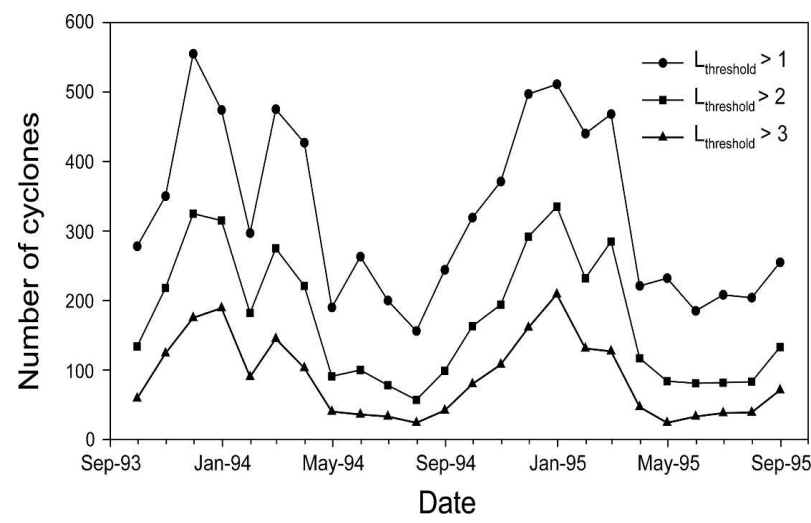

FIG. 5. The monthly number of vortices detected in ECMWF MSL pressure for vortices with $L_{\text {threshold }}$ set to 1,2 , and $3 \mathrm{hPa}$ $(\mathrm{deg})^{-2}$ from October 1993 to September 1995. 

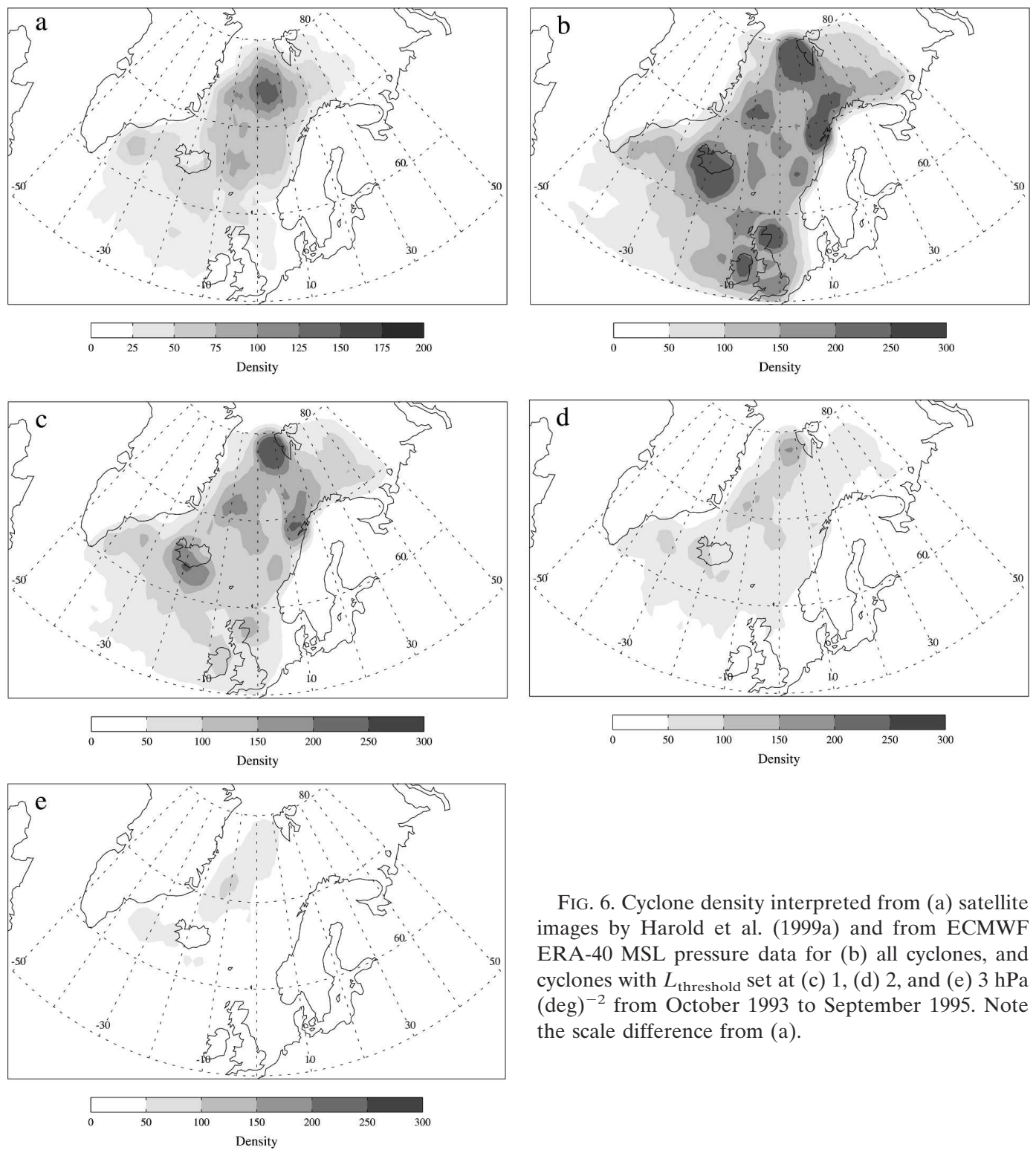

FIG. 6. Cyclone density interpreted from (a) satellite images by Harold et al. (1999a) and from ECMWF ERA-40 MSL pressure data for (b) all cyclones, and cyclones with $L_{\text {threshold }}$ set at (c) 1, (d) 2, and (e) $3 \mathrm{hPa}$ $(\mathrm{deg})^{-2}$ from October 1993 to September 1995. Note the scale difference from (a).

maxima in the MSL pressure field are classed as cyclones [i.e., $L_{\text {threshold }}$ set to $0 \mathrm{hPa}(\mathrm{deg})^{-2}$ ], $70 \%-80 \%$ of cloud vortices with diameters greater than $500 \mathrm{~km}$ are captured in the MSL pressure field data (i.e., they have a pressure signature). However, as the polar mesocyclonic cloud diameter decreases their chances of detection reduces; less than $20 \%$ of cloud systems below 250-km diameter are associated with a cyclone in the reanalysis. When weaker cyclones are screened out our ability to associate a cyclonic circulation to a cloud vortex is reduced even further. Removing cyclones with a Laplacian strength below $2 \mathrm{hPa}(\mathrm{deg})^{-2}$ reduces the chance of associating a cyclonic pressure signature to a cloud system with a diameter below $250 \mathrm{~km}$ to less than

$10 \%$, and $60 \%$ of cloud vortices with a diameter greater than $500 \mathrm{~km}$ are captured.

Figure 8 shows the total number of cyclones detected by the cyclone identification algorithm at MSL pressure against their Laplacian strength. The distribution is skewed to the left with a modal strength of $1-1.5 \mathrm{hPa}$ $(\mathrm{deg})^{-2}$. It is interesting to note that this distribution is somewhat similar to that observed by Harold et al. (1999a; see Fig. 7 for a comparison). Open vortices are skewed farther to the left with a modal strength of 0.5-1 $\mathrm{hPa}(\mathrm{deg})^{-2}$, signifying they are weaker systems (not shown). Screening cyclones by setting $L_{\text {threshold }}$ to $1 \mathrm{hPa}$ $(\mathrm{deg})^{-2}$ removes over 3000 vortices in total, but has the effect of reducing the overall total number of cloud 


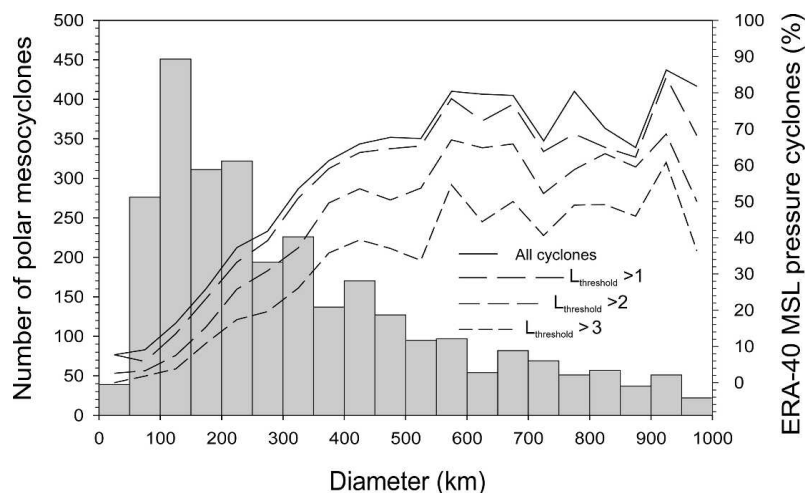

FIG. 7. Number of satellite-observed cloud vortices detected over the 2-yr climatology per 50-km size category (shaded bars). Overlaid are the percentages of cloud vortices in each size group with a cyclonic circulation in the MSL pressure reanalysis for all cyclones and those when $L_{\text {threshold }}$ is set at 1,2 , and $3 \mathrm{hPa}(\mathrm{deg})^{-2}$.

vortices found with a pressure signature by only $4 \%$, from $48 \%$ to $44 \%$ (Table 1 ). The fact that such a good agreement (in terms of the percentage of polar mesocyclonic cloud features with a circulation in the pressure data field) is kept suggests that many of the systems below this strength threshold are likely to be spurious. We shall return to this in the discussion.

\section{b. 500-hPa geopotential height}

At the 500-hPa geopotential height level, a maximum of $35 \%$ of all cloud vortices can be related to a cyclone in the reanalysis data over the 2 -yr period. This was achieved by allowing all maxima in the Laplacian of the 500-hPa geopotential height field to be classed as cyclones (i.e., no weak systems were removed; Table 1). The monthly number of satellite and reanalyzed cyclones show similar variations over the 2-yr period (Fig. 9), with a correlation coefficient of 0.80 that is statisti-

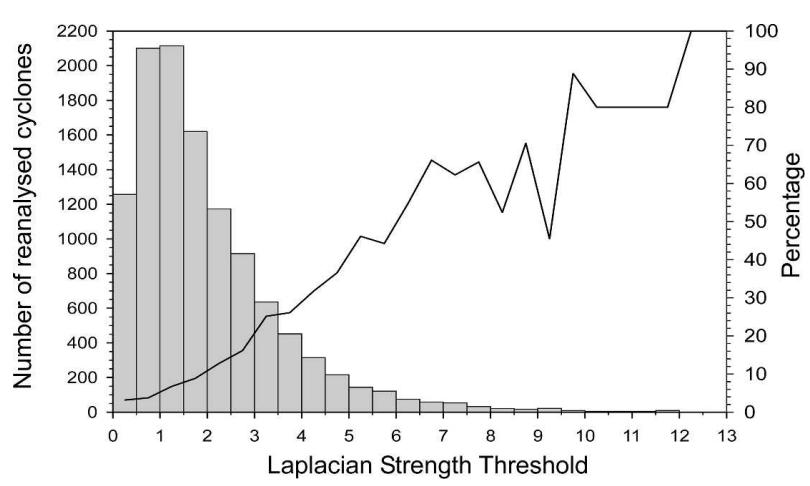

FIG. 8. The number of cyclones found in MSL pressure against cyclone strength as determined by the Laplacian maximum $[\mathrm{hPa}$ $\left.(\mathrm{deg})^{-2}\right]$. The percentage of cyclones for each strength category with a cloud signature is shown by the solid line.
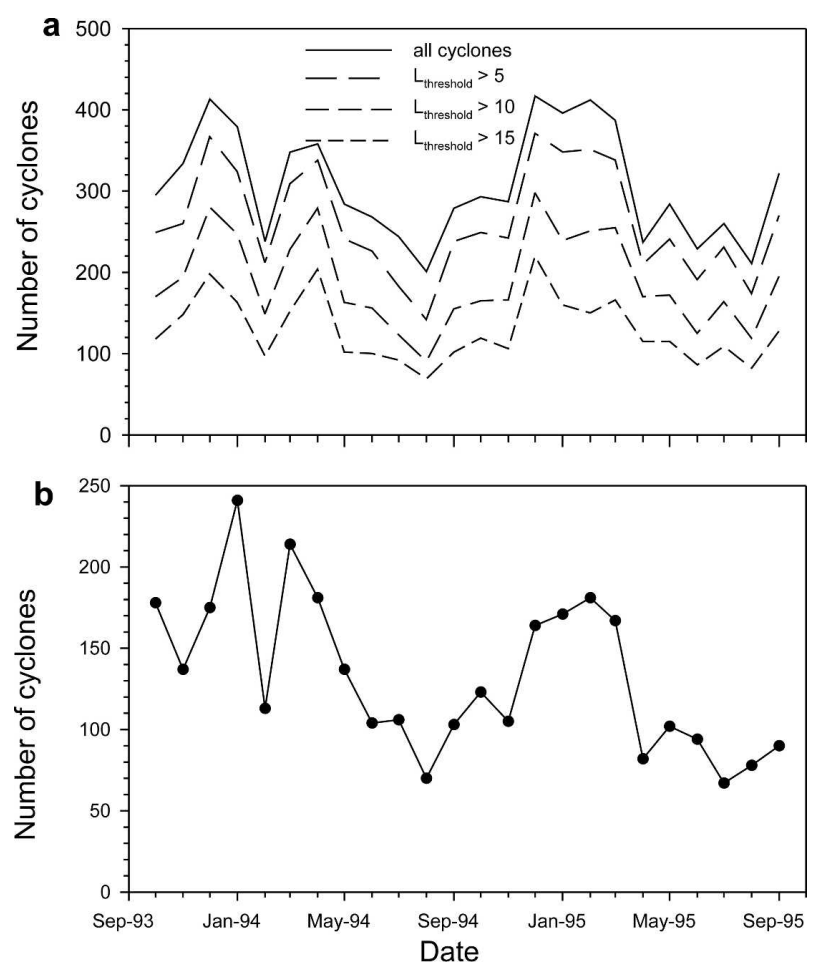

FIG. 9. The number of cyclones detected in 500-hPa geopotential height at (a) different Laplacian strengths and (b) cloud vortices located in satellite imagery (the same as in Fig. 4b).

cally significant at the $99 \%$ confidence interval. The 500-hPa height cyclone time series displays a very similar seasonal cycle to cyclones observed in the MSL pressure field with a peak in cyclogenesis during the winter (an average of 360 cyclones winter $^{-1}$ ), compared to the summer (an average of 253 cyclones winter ${ }^{-1}$ ). Increasing $L_{\text {threshold }}$ reduces the total number of cyclones detected, although there is only a slight reduction in the correlation with the satellite-observed systems (Table 1). As with the trend detected in the MSL pressure data, the monthly number of cyclones detected in the 500-hPa height field shows a decline over the two years (Fig. 9), although it is not found to be statistically significant. Furthermore, an analysis of open and closed systems reveals the decline to again be confined to the open systems, with the number of closed systems remaining fairly constant throughout the 2-yr period (not shown).

\section{1) Cyclone distribution}

With the cyclone identification algorithm at its most sensitive the density distribution reveals the highest cyclone activity to occur between the latitude bands of $20^{\circ} \mathrm{W}$ and $30^{\circ} \mathrm{E}$, where densities are over 120 vortices per $125000 \mathrm{~km}^{2}$ (Fig. 10). An especially high region of 

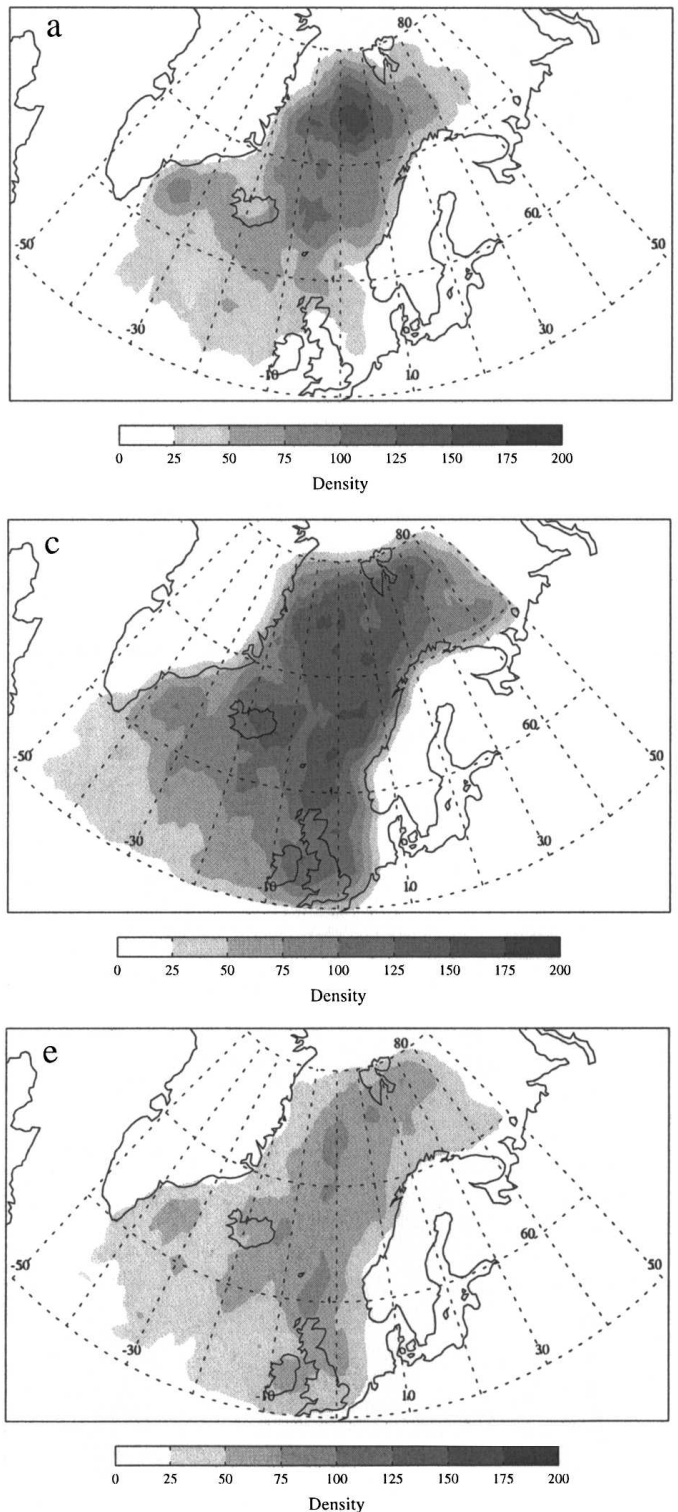

activity is noted from north to south near the $0^{\circ}$ meridional line, along the coast of Norway, over Iceland, and also over much of the Greenland-Norwegian Sea. No obvious small-scale regions of cyclone density maxima are visible in this height field, unlike the case for the MSL pressure systems. The spatial correlation with vortices located in the satellite imagery is high $(r=$ 0.84 ), suggesting cyclones depicted in this height field represent the location of polar mesocyclones observed in the satellite imagery. As we screen out weaker cyclones, the high cyclone density over much of the northeast Atlantic is reduced in comparison to background levels over the region, suggesting these features are fairly indistinct. Removing cyclones with a Laplacian strength below $15 \mathrm{gpm}(\mathrm{deg})^{-2}\left[L_{\text {threshold }}\right.$ equals $15 \mathrm{gpm}$
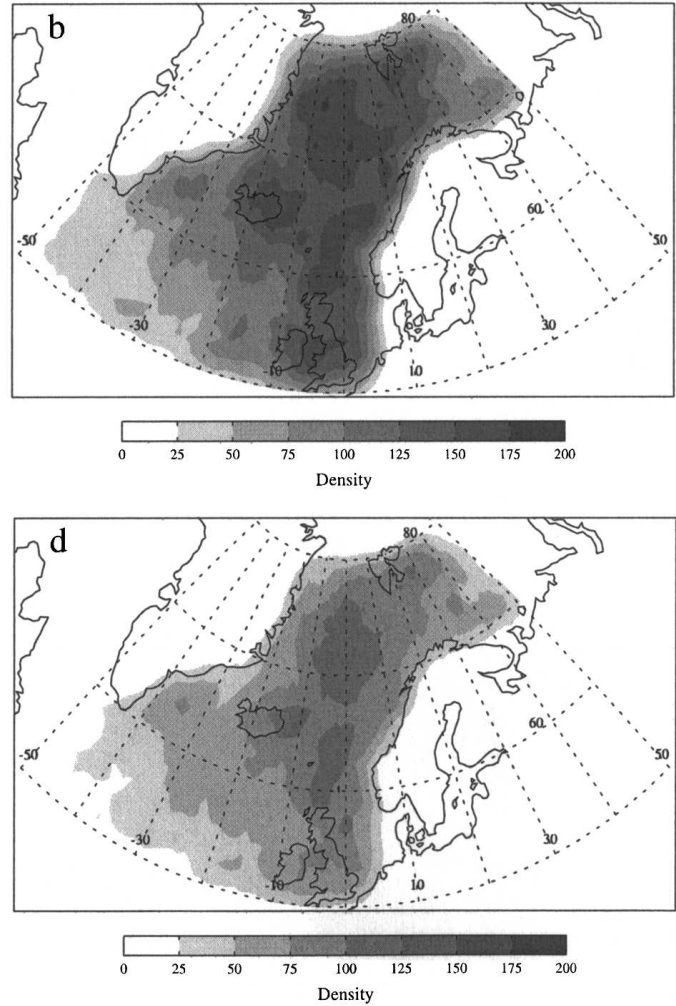

FIG. 10. Cyclone density interpreted from (a) satellite images by Harold et al. (1999a) (the same as in Fig. 6a) and from ECMWF ERA-40 500-hPa geopotential height for (b) all cyclones, and those cyclones with a $L_{\text {threshold }}$ of (c) 5, (d) 10, and (e) $15 \mathrm{gpm}(\mathrm{deg})^{-2}$ from October 1993 to September 1995.

$\left.(\mathrm{deg})^{-2}\right]$ resulted in the closest number match with an overall total of 3101 depressions that is in good agreement with the 3004 cyclones detected in the satellite imagery (Table 1), and little change in the spatial correlation as the sensitivity of the algorithm is changed.

\section{2) Characteristics of Systems FOUnd IN 500-HPA GEOPOTENTIAL HEIGHT}

Larger cloud systems are more frequently found to have a detectable cyclone in the $500-\mathrm{hPa}$ geopotential height (Fig. 11). If all reanalyzed vortices are included, around $60 \%$ of cloud vortices greater than $500 \mathrm{~km}$ in diameters are captured; while on average only $12 \%$ of polar mesocyclones below $250-\mathrm{km}$ diameter have a signature in the reanalysis data field. This result is in 


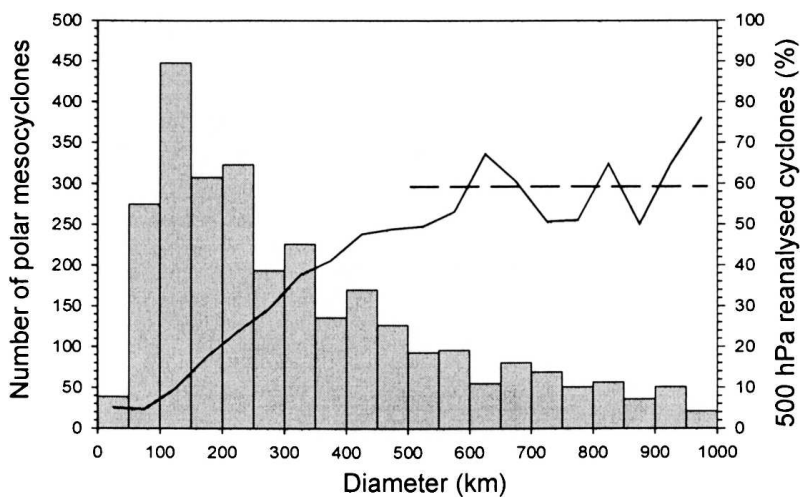

FIG. 11. Number of satellite-observed cloud vortices detected over the 2-yr climatology per 50-km size category (shaded bars). Overlaid are the percentages of cloud vortices in each size group with a cyclonic circulation in the 500-hPa height level for all cyclones. The dotted line shows the average number of vortices with a cloud feature above 500-km diameter.

agreement with what was found for systems in the MSL pressure (Fig. 7). We discuss the question of the vertical extent of systems and their correspondence with the satellite climatology further in the next section.

\section{Discussion}

Variations in monthly vortex numbers from October 1993 to September 1995 detected in both the ERA-40 MSL pressure and 500-hPa geopotential height field using the Murray and Simmonds (1991) detection algorithm show a very similar trend to that observed in satellite imagery by Harold et al. (1999a). During the winter both the derived reanalysis climatologies and the satellite cloud climatology show a peak in mesocyclone activity, with fewer cyclones recorded in the summer. This is consistent with existing satellite observations of polar mesocyclonic activity (Wilhelmsen 1985; Lystad 1986).

Accepting all Laplacian maxima as cyclones in the reanalysis data gives the highest chance of associating a cloud system in the satellite climatology with a pressure signature in the data fields analyzed; $48 \%$ and $35 \%$ of all cloud vortices could be associated with MSL pressure and 500-hPa reanalyzed cyclones, respectively. Of those vortices detected on the satellite imagery with a surface signature in the reanalysis data, $60 \%$ also have an upper-level circulation at the 500-hPa geopotential height level, suggesting these vortices have a definite three-dimensional structure. These systems are typically larger in size, with a modal diameter of 400-450 $\mathrm{km}$ and mean Laplacian strength of $4.0 \mathrm{hPa}(\mathrm{deg})^{-2}$, compared to a modal diameter of $300-350 \mathrm{~km}$ and a mean Laplacian strength of $3.3 \mathrm{hPa}(\mathrm{deg})^{-2}$ for all cloud vortices with a MSL pressure signature. On average, $34 \%$ of cloud vortices with a detectable circulation in ERA-40 were vertically restricted to the lower atmosphere, having no detectable circulation at the 500-hPa level. These cyclones are, on average, small and weak with a mean Laplacian strength of $2.7 \mathrm{hPa}$ $(\mathrm{deg})^{-2}$ and modal diameter of $200-250 \mathrm{~km}$. The remaining small fraction $(6 \%)$ of the cloud vortices with a detectable circulation in ERA-40 were confined to the 500-hPa geopotential height level, showing no detectable signature in the MSL pressure field, and consequently will have little impact upon the underlying ocean.

For cyclones detected in the ERA-40 reanalysis at both pressure levels, on average $54 \%$ of mesocyclonic cloud features observed on the satellite imagery have a detectable circulation. This observation is consistent with Harold et al. (1999b), who determined that if a surface circulation existed for vortices crossing Ocean Weather Ship Mike in the Norwegian Sea $\left(66^{\circ} \mathrm{N}, 2^{\circ} \mathrm{E}\right)$ depending on whether or not they displayed a pressure minimum in the data logged at the weather station. Despite the different detection method, Harold et al. (1999b) concluded that $54 \%$ of mesocyclonic cloud patterns had a surface circulation, which is in strong agreement with our observations.

The extremely high numbers of vortices identified in the reanalysis when all maxima in the Laplacian of the pressure field are considered to be cyclones is likely due to the detection of a significant number of weak vortices in regions of slack pressure (Murray and Simmonds 1991; Sinclair 1994). When weaker cyclones are screened out by increasing the Laplacian threshold strength, our ability to associate a cloud vortex with a reanalyzed cyclone reduces. However, the removal of cyclones detected in MSL pressure when the $L_{\text {threshold }}$ was set to $1 \mathrm{hPa}(\mathrm{deg})^{-2}$ resulted in the removal of over 3000 vortices, yet only reduced the overall total number of cloud vortices with a detectable circulation signature by $4 \%$ This finding suggests that many vortices detected in the reanalysis below $1 \mathrm{hPa}(\mathrm{deg})^{-2}$ are likely spurious, and do not represent true mesocyclones. Although it could be argued that there are in fact many more mesocyclones detected in the reanalysis data than were observed from the satellite imagery, the weakest features in the reanalysis are seldom associated with a cloud pattern suggesting they are an artifact of the detection algorithm and are of no consequence to the underlying ocean. In short, setting $L_{\text {threshold }}$ to 1 or 2 $\mathrm{hPa}(\mathrm{deg})^{-2}$ provides the most appropriate picture of surface cyclone activity in the reanalysis data.

Our ability to detect polar mesocyclones in the reanalysis pressure data fields is strongly influenced by 
the size of the system, with larger cloud vortices more likely to have a circulation. Of the 3004 cyclonic cloud patterns, $70 \%-80 \%$ of cloud systems with a diameter greater than $500 \mathrm{~km}$ are detected in the MSL pressure field but represent only $25 \%$ of the total number of observed mesocyclones, while $50 \%$ of the satelliteobserved cloud systems have a diameter less than 250 $\mathrm{km}$, yet on average only $20 \%$ of these systems are detected in the MSL pressure reanalysis data. Hence, a larger number of mesocyclonic vortices are in the size group most poorly represented in the reanalysis. A similar pattern is observed for cyclones in the $500-\mathrm{hPa}$ geopotential height level, in which cloud vortices with a diameter greater than $500 \mathrm{~km}$ are consistently detected around $60 \%$ of the time in the reanalysis. The lower detection of cloud vortices in the 500-hPa geopotential height field compared to MSL pressure has been shown to be associated with smaller, weaker mesocyclonic cloud systems being vertically restricted and therefore not possessing a resolvable circulation higher in the atmosphere.

In a study of mesoscale vortices over the Antarctic, Marshall and Turner (1997) examined the circulation of polar mesocyclones detected in AVHRR satellite data using scatterometer data. Although the sizes of the different systems inspected are not given by the authors, the percentage of mesocyclones observed in satellite imagery that were resolved in scatterometer data was $75 \%$. This observation is the same as we observed for polar mesocyclones greater than $500 \mathrm{~km}$ in diameter in the ERA-40 MSL pressure reanalysis, and is in agreement with Marshall and Turner (1997) that around 25\% of mesoscale cloud patterns do not appear to have a detectable cyclonic circulation.

The inability of the reanalysis to assimilate polar mesocyclones as they decrease in size is also in agreement with Turner and Thomas (1994), who observed that the Meteorological Office's coarse $(150 \mathrm{~km})$ mesh, global model analysis scheme had only limited success at representing mesoscale vortices found in satellite imagery over the Antarctic. It was shown that polar mesocyclones identified on satellite imagery greater than 500 $\mathrm{km}$ in diameter appeared in the model analysis $44 \%$ of the time while polar mesocyclones with a diameter between 200 and $500 \mathrm{~km}$ were identified $16 \%$ of the time, and those less than $200 \mathrm{~km}$ in diameter only appeared in the model analysis $2 \%$ of the time. Although the correct identification of mesocyclones observed in satellite imagery around Antarctic is lower than observed by us for the ERA-40 reanalysis, there is a clear bias against resolving polar mesocyclones as they decrease in size. The lower observations around Antarctica may be a result of reduced amounts of in situ data being assimilated into the model in this region compared to the North Atlantic. The higher model resolution in ERA-40 may also be able to better resolve mesoscale systems.

ERA-40 appears unable to resolve a substantial proportion of polar mesocyclones with a diameter less than $500 \mathrm{~km}$. The consistent detection of $\sim 80 \%$ of the cloud features with a diameter greater than $500 \mathrm{~km}$ is in agreement with the requirement of four (or more) grid points suggested by Pielke (1991) (considering the eastwest reanalysis grid length of $\sim 125 \mathrm{~km})$. However, as noted in section 2 , the smallest resolvable feature in a spectral model is given by the spectral truncation limit (in this case T159); thus, cyclones with a diameter down to $125 \mathrm{~km}$ could be captured. The reduction in cyclone detection below $500 \mathrm{~km}$ suggests that ERA-40 can represent features of scales less than $500 \mathrm{~km}$, but only if they are sufficiently sharp or well placed within the grid.

Furthermore, as polar mesocyclones are relatively short lived (3-36 h) they may not always be assimilated into the reanalysis due to a lack of in situ observations, especially the most rapidly forming and transient vortices that will only be present for one or two reanalysis periods. Harold et al. (1999b) noted that the mesocyclones in the northeast Atlantic with diameters less than $400 \mathrm{~km}$ in general had much shorter lifetimes compared with larger mesocyclones with diameters 400$1000 \mathrm{~km}$, often lasting less than $15 \mathrm{~h}$. Hence, their short lifetime may have caused a large fraction of the small systems to not be assimilated into the reanalysis, and may account for some of the decline in the detection of mesocyclones with a diameter less than $500 \mathrm{~km}$ in ERA-40.

Based on the high number of satellite cloud vortices with a diameter less than $500 \mathrm{~km}$ observed without any surface circulation in the reanalysis MSL pressure field, ocean models forced with the present ERA-40 reanalysis surface field are likely to significantly underrepresent air-sea fluxes of heat, moisture, and momentum. The importance of accurately representing mesoscale features in models was highlighted by Pickart et al. (2003), who forced a regional ocean model with a representation of the Greenland tip jet in which an accurate satellite-derived estimate of the tip-jet wind stress curl and heat flux were applied, rather than these fluxes being taken from reanalysis data. A region of increased surface density, roughly $200 \mathrm{~km}$ in diameter, formed to the east of Cape Farewell, with a mixed layer extending to $2000 \mathrm{~m}$, representing enhanced open-ocean convection and deep-water formation. When only the coarse reanalysis fluxes are used such vigorous convection was not simulated, thus providing evidence that these me- 
soscale atmospheric features must be represented somehow for accurate ocean modeling. We would suggest that vortices with a diameter less than $500 \mathrm{~km}$ must be parameterized in order to accurately represent heat, moisture, and momentum fluxes, especially over the Greenland-Norwegian Sea where heat loss to the atmosphere is important for the sinking of deep water and determining thermohaline circulation stability. This topic will form the subject of a future paper in which we will examine how an ocean-only climate model forced with the heat, moisture, and momentum fluxes of polar mesocyclones below $500 \mathrm{~km}$ in diameter missing from ERA-40 alters the intensity of the thermohaline circulation.

The spatial correlation between satellite-observed polar mesocyclones and vortices in both the MSL pressure and $500-\mathrm{hPa}$ height reanalysis is generally very good, with correlation coefficients around 0.8 (Table 1), suggesting that the general pattern of cyclone representation in both fields is very similar. We do however note the tendency of cyclones to cluster in specific regions in the MSL pressure field when all maxima in the Laplacian of the pressure are accepted as cyclones, as indicated by the eight cyclonic maxima in the northeast Atlantic region. The absence of any cyclonic maxima in the $500-\mathrm{hPa}$ geopotential height level suggests that these features are confined to the low level. The cyclonic maxima appear to line up with the principal synoptic weather stations in the northeast Atlantic (Fig. 12). Unfortunately, a list of synoptic stations routinely used in the ECMWF ERA-40 reanalysis was not available to us, so instead we use stations listed on the Europe Synoptic Stations dataset produced by the Meteorological Office and made available to us through the British Atmospheric Data Center. All of these stations routinely make upper-level measurements through radiosonde release, and are also listed as making daily or near-daily observations in the ECMWF quarterly statistics at the $10-\mathrm{hPa}$ height level. As such, we believe surface observations from these locations are likely to be routinely assimilated into the ERA-40 reanalysis. A two-tailed $t$ test is used to determine whether the high density in the vicinity of the weather stations is simply due to chance, or is an artifact of observations being taken from these regions. All of the data grid points of cyclone density that are within $200 \mathrm{~km}$ of each weather station are statistically compared to the cyclone density at the same number of randomly selected gridpoint locations (excluding land) over the northeast Atlantic. Cyclone densities surrounding the synoptic weather stations are found to be statistically different (above the 95\% confidence interval) from the random locations selected, indicating that it is not by chance that higher

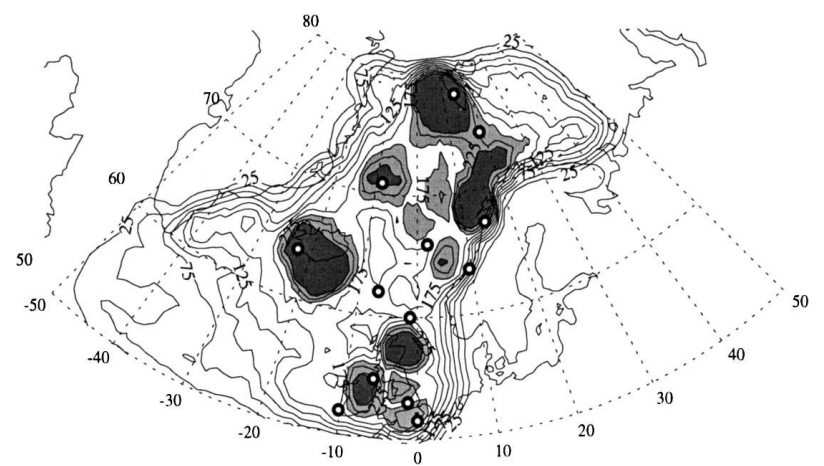

FIG. 12. Principal synoptic weather stations (shown by a black and white circle) located over the northeast Atlantic, and cyclone density per $125000 \mathrm{~km}^{2}$ for all cyclones detectable in the ERA-40 MSL pressure data field, i.e., no Laplacian strength threshold is imposed, as in Fig. 6b. The contour interval is 25 , and values greater than 175 (225) are shaded (heavily).

cyclonic densities are observed in these locations, but that they appear to be associated with the presence of the weather stations. The persistence of these features was tested by screening out weaker cyclones. It was noted that when $L_{\text {threshold }}$ was set to $3 \mathrm{hPa}(\mathrm{deg})^{-2}$ these vortices were removed from the MSL pressure data. Furthermore, a comparison of cyclone density maps of both open and closed lows (not shown), revealed the maxima to be restricted to the weaker open systems. We conclude that these features are fairly weak. A separate analysis of $30 \mathrm{MSL}$ reanalysis pressure charts showing the locations of vortices located using the cyclone algorithm for January 1994 over the northeast Atlantic (not shown) confirmed this finding. Stationary vortices were frequently observed in the regions of the weather stations, and often in regions of rather slack pressure gradient. Thus, we believe the vortices repeatedly detected in the vicinity of the weather station are likely to be spurious. The maxima appear most pronounced over weather stations not fully surrounded by ocean, and we suggest that observations from such stations may be more representative of the neighboring land than the surrounding ocean. We suggest that there may be a problem of the representativeness of the MSL pressure data in the ERA-40 reanalysis in these locations. The assimilation problem has the effect of creating small minima in the pressure field, which are subsequently interpreted as depressions by the cyclone detection algorithm, and as such are contributing to the more than 3000 weak reanalyzed vortices observed below $1 \mathrm{hPa}(\mathrm{deg})^{-2}$ that are not associated with a mesocyclonic cloud pattern. We also suspect that the overrepresentation of cyclone activity over weather stations will occur for any algorithm attempting to detect small weak mesocyclones as vorticity maxima from this data. 
Finally we observe that the satellite, MSL pressure, and 500-hPa geopotential height climatologies all reveal a decline in cyclonic activity over the 2-yr period. Harold et al. (1999a) noted that the decline was mainly confined to a reduction in the number of small shortlived cloud vortices, and this is reiterated by the sharp decline in cloud features with sizes up to $500 \mathrm{~km}$ in diameter, while the number of vortices with a diameter of 500-1000 km remained fairly constant over the 2-yr period (Fig. 4). The analysis of both open and closed lows from the ERA-40 data supports the observed reduction, with the decline being confined to the open (i.e., weaker) systems, while the number of closed lows remained fairly constant over the 2 -yr period. The open lows represent the smaller subsynoptic systems compared to the closed lows, as shown by their modal class sizes of $150-$ and $450-\mathrm{km}$ diameter, respectively, for MSL pressure reanalyzed vortices that were associated with cloud systems. Thus, the reanalysis data supports the observation by Harold et al. (1999a) that the number of small systems declined over the 2-yr period. The high interannual variability in mesocyclone numbers and the relatively short 2-yr comparison period makes it difficult to infer whether the observed decline is part of a long-term reduction in mesocyclone activity. We suggest, however, that polar mesocyclones are likely to be captured to the same extent in the reanalysis data outside of the 2-yr comparison period and so, in future work, plan to examine the variation in mesocyclone numbers through the full 40-yr ERA-40 dataset.

\section{Summary and conclusions}

Using a combination of the MSL pressure and 500hPa geopotential height fields, $54 \%$ of cyclonic cloud vortices can be associated with a circulation signature in the ERA-40 reanalysis. Considering the significant number of cloud vortices missing from the reanalysis below $\sim 500 \mathrm{~km}$ in diameter, our results suggest that a considerable amount of heat, moisture, and momentum flux at the air-sea interface is likely to be missing, thus presenting a serious drawback when using such reanalysis data to force ocean models. Hence, we suggest that there is a need for a parameterization of mesoscale cyclone activity below $500 \mathrm{~km}$, if we are to correctly model how the atmosphere affects rates of deep-water formation and the climate system. This shortcoming is not only relevant for forcing ocean models, but valid for many other applications of the ERA-40 dataset.

There is a tendency for cyclones detected in the MSL pressure field to be clustered over synoptic observing stations in the northeast Atlantic region, suggesting there may be a subtle problem in the data assimilation procedure in the ERA-40 reanalysis. These clusters appear most pronounced over weather stations that are not fully surrounded by ocean, leading us to suggest that observations from these sites may be more representative of the land than the surrounding ocean. When higher Laplacian thresholds are used in the detection algorithm, these clusters are removed and the spatial pattern of cyclone density corresponds closely to that of the satellite-derived climatology.

There are very similar trends in monthly cyclone numbers in both the ERA-40 MSL pressure and 500$\mathrm{hPa}$ geopotential height data, and those observed in satellite imagery (by Harold et al. 1999a). There is a decline in the number of smaller (weaker) systems in the northeast Atlantic over the 2-yr period in both the reanalysis data and the satellite climatology. The relatively short period of comparison makes it difficult to infer whether the observed decline is part of a longerterm reduction in mesocyclone activity, suggesting that an extension of the comparison would be beneficial.

Acknowledgments. This work was carried out under an NERC-funded studentship tied to the COAPEC thematic program, and a CASE studentship award from the British Antarctic Survey. The authors thank the British Antarctic Survey for providing access to the ECMWF ERA-40 data, and for assistance with the tracking scheme from Tom Lachlan-Cope. We would also like to thank Andrew Brooks at the NERC Satellite Receiving Station at Dundee University (Dundee, United Kingdom) who very kindly removed depressions located in the reanalysis pressure data outside of each corresponding satellite pass.

\section{REFERENCES}

Businger, S., 1985: The synoptic climatology of polar low outbreaks. Tellus, 37A, 419-432.

- 1987: The synoptic climatology of polar-low outbreaks over the Gulf of Alaska and the Bering Sea. Tellus, 39A, 307-325.

_ , and R. Reed, 1989: Cyclogenesis in cold air masses. Wea. Forecasting, 4, 133-156.

Carleton, A. M., 1985: Satellite climatological aspect of the "polar low" and "instant occlusion." Tellus, 37A, 433-450.

_ _ and D. A. Carpenter, 1990: Satellite climatology of "Polar Lows" and the broadscale climatic associations for the southern hemisphere. Int. J. Climatol., 10, 219-246.

Hakkinen, S., 1995: Simulated interannual variability of the Greenland Sea deep water formation and its connection to surface forcing. J. Geophys. Res., 100, 4761-4770.

Harold, J. M., G. R. Bigg, and J. Turner, 1999a: Mesocyclone activity over the northeast Atlantic. Part 1: Vortex distribution and variability. Int. J. Climatol., 19, 1187-1204.

,-- , and $\longrightarrow$ 1999b: Mesocyclone activity over the northeast Atlantic. Part 2: An investigation of the causal mechanisms. Int. J. Climatol., 19, 1283-1299.

Hewson, T. D., G. C. Craig, and C. Claud, 1995: A polar low 
outbreak. Evolution and Mesoscale Structures, Joint Centre for Mesoscale Meteorology, Internal Rep. 45, 36 pp.

Laprise, R., 1992: The resolution of global spectral models. Bull. Amer. Meteor. Soc., 73, 1453-1454.

Leonard, S. R., J. Turner, and A. van der Wal, 1999: An assessment of three automatic depression tracking schemes. $\mathrm{Me}$ teor. Appl., 6, 173-183.

Lystad, M., 1986: Polar lows in the Norwegian, Greenland and Barents Sea. Final Report, Norwegian Meteorological Institute, $196 \mathrm{pp}$.

Marshall, G. J., and J. Turner, 1997: Surface wind fields of Antarctic mesocyclones derived from ERS 1 scatterometer data. J. Geophys. Res., 102, 13 907-13 921.

Marshall, J., and F. Schott, 1999: Open ocean convection: Observations, theory and models. Rev. Geophys., 37, 1-64.

Moore, G. W. K., M. C. Reader, J. York, and S. Sathiyamoorthy, 1996: Polar lows in the Labrador Sea-A case study. Tellus, 48A, 17-40.

Murray, R. J., and I. Simmonds, 1991: A numerical scheme for tracking cyclone centres from digital data. Part I: Development and operation of the scheme. Aust. Meteor. Mag., 39, $155-166$.

Nordeng, T. E., and E. A. Rasmussen, 1992: A most beautiful polar low: A case study of a polar low development in the Bear Island region. Tellus, 44A, 81-99.

Pickart, R. S., M. A. Spall, M. H. Ribergaard, G. W. K. Moore, and R. F. Milliff, 2003: Deep convection in the Irminger Sea forced by the Greenland tip jet. Nature, 424, 152-156.

Pielke, R. A., 1991: A recommended specific definition of "resolution." Bull. Amer. Meteor. Soc., 72, 1914.

Rasmussen, E. A., 1985: A case study of a polar low development over the Barents Sea. Tellus, 37A, 407-418.

, and J. Turner, 2003: Polar Lows: Mesoscale Weather Systems in the Polar Regions. Cambridge University Press, 612 pp.
— C. Claud, and J. F. Purdom, 1996: Labrador Sea polar lows. Global Atmos.-Ocean Syst., 4, 275-333.

Renfrew, I. A., 2003: Polar lows. Encyclopedia of the Atmospheric Sciences, J. Holton, J. Pyle, and J. Curry, Eds., Georgia Institute of Technology, 1761-1768.

Shapiro, M. A., L. S. Fedor, and T. Hampel, 1987: Researching aircraft measurements of a polar low over the Norwegian Sea. Tellus, 39A, 272-306.

Shaw, G., and D. Wheeler, 2000: Statistical Techniques in Geographical Analysis. David Fulton Publishers, 359 pp.

Simmonds, I., R. J. Murray, and R. M. Leighton, 1999: A refinement of cyclone tracking methods with data from FROST. Aust. Meteor. Mag., June, 35-49.

Simmons, A. J., and J. K. Gibson, Eds., 2000: The ERA-40 Project Plan. ERA-40 Project Report Series 1, 63 pp. [Available online at http://www.ecmwf.int/publications/library/ ecpublications/_pdf/era40/ERA40_PRS_1.pdf.]

Sinclair, M. R., 1994: An objective cyclone climatology for the Southern Hemisphere. Mon. Wea. Rev., 122, 2239-2256.

_ 1997: Objective identification of cyclones and their circulation intensity, and climatology. Wea. Forecasting, 12, 595-612.

Terry, J., and R. Atlas, 1996: Objective cyclone tracking and its applications to ERS-1 scatterometer forecast impact studies. Preprints, 15th Conf. on Weather Analysis and Forecasting, Norfolk, VA, Amer. Meteor. Soc., 146-149.

Turner, J., and J. P. Thomas, 1994: Summer-season Mesoscale cyclones in the Bellingshausen-Weddell Region of the Antarctic and links with the synoptic-scale environment. Int. J. Climatol., 14, 871-894.

Wadhams, P., J. Holfort, E. Hansen, and J. P. Wilkinson, 2002: A deep convective chimney in the winter Greenland Sea. Geophys. Res. Lett., 29, 76-80.

Wilhelmsen, K., 1985: Climatological study of gale-producing polar lows near Norway. Tellus, 37A, 451-459. 